\title{
Complexity in Economic Theory and Real Economic Life
}

\author{
H A N S - WA LTER LORENZ \\ Friedrich-Schiller-Universität Jena, Germany
}

\begin{abstract}
Complex dynamic behaviour in terms of chaotic motion, catastrophic events or other seemingly irregular and unexpected features of and in theoretical economic models - aimed at describing real-world phenomena - are nowadays known as a common property of many nonlinear approaches to an understanding of the motion of actual time series, such as inflation rates, unemployment figures, and many other - mainly macroeconomic - economic variables. Since most existing models in economic dynamics are constructed in the tradition of classical mechanics, this result does not appear as a real surprise. However, the real 'complexity challenge' for economic theory still persists in identifying the complex structure of economic reality, which cannot be satisfactorily represented by simple deterministic laws of motion, although such 'laws' might possess the possibility of very complicated dynamic motion.
\end{abstract}

\section{Introduction}

The term 'complexity' has been one of the most intensively discussed expressions in many scientific disciplines during the last, say, 20 years. Attempts have been made to distinguish between 'complex' and 'complicated' phenomena and to identify the characteristics of 'regular' events (cf. Holland ${ }^{1}$ for a brief introduction to this topic). In the current paper, the occasionally sophisticated discussion documented in the literature will not be followed. Instead, a rather pragmatic distinction will be chosen in order to demonstrate what 'complexity' might mean in existing theoretic models and in actual economic life, which should be modelled, understood and predicted.

The first variant - for example, complexity in the broad sense of irregular motion in a well-defined dynamical system - will be called 'complexity in theoretical economics' (CTE). Research in this field investigates the behaviour of economic dynamical systems constructed out of the conviction that the behaviour of economic agents such as households and firms can be represented by well-defined functional 
relations such as utility functions and production functions on the household, respectively the firm, side of a particular market or even the entire economy. The open and relevant question then deals with the problem of whether these well-defined systems display 'simple' regular dynamic behaviour, like divergence from/convergence to fixed points or simple closed orbits, or, alternatively, display other, more 'complicated' patterns. This research into CET models is - as far as the methodological aspects are concerned - comparable with the mathematical treatment of established dynamical systems in the physical sciences. Occasionally, identical dynamical systems exist in physics and theoretical dynamical economics in the form of van-der-Pol equations, pendulum equations, variants of Lorenz-type equations, Henon-type equations etc. It cannot be doubted that theoretical dynamical economics has adopted the techniques used in parts of the theoretical physical sciences in order to investigate the dynamic properties of these economic models.

CTE models (as well as all theoretical models in economics that can be represented in a mathematical form) have been constructed under at least two premises. Most modern authors are certainly convinced that actual economic life is characterized by an infinite number of facets and varieties and depends on a multitude of influences from other than economic spheres and that it is impossible to grasp an economic phenomenon with a few functional dependencies and structural equations. However, most parts of economic theory (including the work of most recent authors) declare that it is necessary (and sufficient) to abstract from non-economic influences at least to some degree and to concentrate on the most relevant 'pure' economic scenarios. While a few classical authors such as Menger $^{2}$ were convinced that their simple mathematical economic models represented 'real' economic life, abstraction from seemingly rather irrelevant influences is typical for modern economic theory. However, the question is still which (perhaps drastic) errors in the description of actual economic life are being made when economic theory operates on a very high abstraction level. Taking this question seriously, economists are then asked to determine which simplifying assumptions in the description of real economic life are candidates for distorting the results. The consideration of a possible 'complexity in real economic life' (CREL) should desirably also end up in closed mathematical models (whose dynamic behaviour should then be investigated as in CTE models) but - due its nature - more than a listing of potential influences dropped during the abstraction procedure mentioned above cannot be expected.

The paper is organized as follows. The next section provides a very short survey of traditional modelling in economic theory and contains an example of the CTE phenomenon in dynamic economic models. The section also contains a few remarks on the empirical relevance of chaotic motion in real time series. The third section lists several ignored aspects, which appear to be significant in a proper modelling of dynamic economic phenomena, including general 
feedback effects in economic life, bounded rationality, and a dimension problem in the face of evolving economies. A few concluding remarks can be found in the final section.

\section{Complexity in theoretical economic models}

Economic theory - developed as a more or less fully fledged discipline by authors such as, Léon Walras, ${ }^{3}$ Edgeworth, ${ }^{4}$ Jevons, $^{5}$ Menger $^{2}$ or Marshall ${ }^{6}$ during the last decades of the 19th century - can be considered as an inheritor of the scientific progress of the day. Physics - and especially classical mechanics and astronomy represented scientific idols that described fruitful modelling alternatives to the traditional philosophically oriented approaches in the 'soft' sciences (including economics, regional development studies, and the emerging theoretical sociology), which were characterized by lengthy verbal reflections for decades.

Classical authors - if they were concerned with dynamic problems at all concentrated on the stability of equilibria (in the economic sense of a fitting of supply and demand at certain prices). However, the recent economic literature actually abounds with examples of the potential emergence of complex motion in one-dimensional and two-dimensional discrete-time dynamical systems. As a rule of thumb, complex motion can almost always be observed (for appropriate parameter values) in discrete-time, two-dimensional systems that are derived from originally continuous-time systems (permitting limit-cycle behaviour) by substituting the differential operator by finite differences. Economic examples of complicated motion in 3D continuous time models exist but are rare.

The following example is due to Herrmann ${ }^{7}$ who studied a two-dimensional, discrete-time business-cycle model with Kaldorian elements (coined after $\mathrm{N}$. Kaldor, a renowned economist during Cambridge's prime in economic theory in the 1930s and 1940s):

$$
\begin{aligned}
\Delta Y_{t+1} & =\alpha\left(I\left(Y_{t}, K_{t}\right)+C\left(Y_{t}\right)-Y_{t}\right) \\
\Delta K_{t+1} & =I\left(Y_{t}, K_{t}\right)-\delta K_{t}
\end{aligned}
$$

with $Y_{t}$ as income in period $t, I(\cdot)$ as (gross) investment, $C(\cdot)$ as consumption, and $K_{t}$ as the (real) capital stock in a closed economy. The coefficient $\alpha>0$ is an adjustment parameter, $\delta>0$ represents the depreciation rate in this economy. The first equation states that the change in income from period $t$ to period $t+1$ depends on the difference between demand, i.e. $I(\cdot)+C(\cdot)$, and income $Y$ in every period; the second equation represents a definition, namely that the change in the capital stock is defined as the difference between gross investment and the amount of depreciation in each period.

Assume that $I\left(Y_{t}, K_{t}\right)=\beta\left(K_{t}^{d}-K_{t}\right)+\delta K_{t}$, i.e. gross investment depends proportionally on possible discrepancies between the desired capital stock, $K_{t}^{d}$, and 
the actual capital stock, $K_{t}$. If the desired capital stock depends linearly on output, i.e. $K_{t}^{d}=k Y_{t}, k>0$ equations (1) turn into

$$
\begin{aligned}
\Delta Y_{t+1} & =\alpha\left(\beta\left(k Y_{t}-K_{t}\right)+\delta K_{t}+C\left(Y_{t}\right)-Y_{t}\right) \\
\Delta K_{t+1} & =\beta\left(k Y_{t}-K_{t}\right)
\end{aligned}
$$

or

$$
\begin{aligned}
Y_{t+1} & =\alpha\left(\beta\left(k Y_{t}-K_{t}\right)+\delta K_{t}+C\left(Y_{t}\right)-Y_{t}\right)+Y_{t} \\
K_{t+1} & =\beta\left(k Y_{t}-K_{t}\right)-(1-\delta) K_{t}
\end{aligned}
$$

For a certain specification of the functional form of the consumption function $C(Y)$ with a sigmoid shape in $C_{t}, Y_{t}$-space and an appropriate parameter set, Herrmann $^{7}$ was able to demonstrate the existence of a strange attractor for the Kaldorian-type model (equation (3)) (details can be found in Refs 7 and 8). In particular, it can be shown that the model (3) possesses a so-called snap-back repeller, i.e. the discrete-time analogue of a homoclinic orbit known from continuous-time dynamical systems. The result for a specific parameter set is shown in Figure 1 (transients are excluded).

The time series of $Y_{t}$ and $K_{t}$ values with a length of about 50,000 points depicted in Figure 1 possess many characteristics used to describe an object as a 'strange attractor' in the literature. In particular, the series has a positive largest Lyapunov exponent and the correlation dimension is a fractal between 1 and 2, indicating that the object in Figure 1 is indeed a strange (or 'chaotic') attractor.

The object in Figure 1 persists under small perturbations of the model and variations in the parameter set. However, it should be recalled that the result of any numerical example cannot be generalized to hold true fort the entire range of

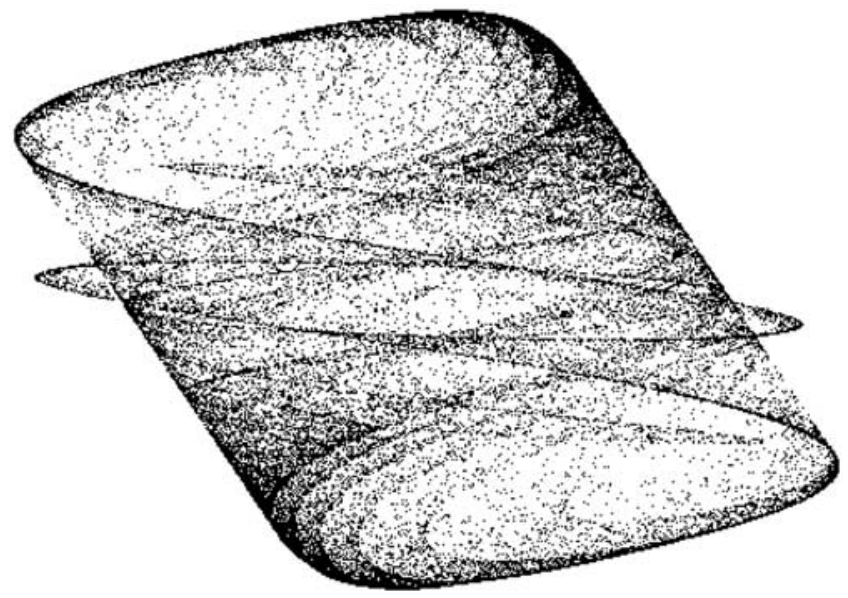

Figure 1. Chaos in a discrete-time Kaldor model (Y [horizontal] - versus K [vertical]) 


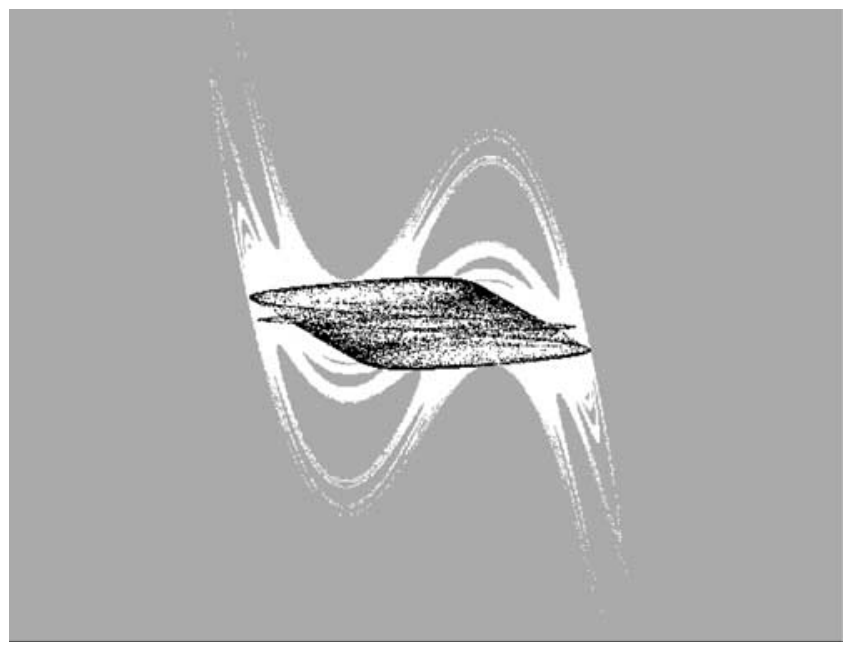

Figure 2. A basin of attraction of equation (3) (white area) (Y vs. K)

parameter values in a certain model. In fact, a separate numerical study is required for each numerical specification of a model.

While the object in Figure 1 was called an 'attractor', it should be noted that the basin of attraction is rather limited. This basin is depicted in Figure 2. Initial values, which are attracted by the black object (the 'attractor'), are represented by points in the white area. Initial values in the grey region tend towards $\pm \infty$. Since the divergence area comes very close to the attractor it is by no means obvious (without a precise knowledge of the attractor region) whether an arbitrary initial value converges to the attractor or not.

The separate plotting of the time series $Y_{t}$ and $K_{t}$ versus time shows the irregularity as well as the sensitive dependence on initial conditions (SDIC) more clearly. In Figure 3, the time series of $Y_{t}$ (upper plot) and of $K_{t}$ (lower plot) are shown for slightly different initial values. After wandering together for a few periods, the two time series eventually diverge. This property of the system prevails when higher $t$-values are considered, i.e. the phenomenon is not restricted to the transient phase.

Of course, it is tempting to consider even this simple example of SDIC as a manifestation of the often expressed conviction that it is impossible to provide proper projections of the recent development of an economy into the future by economic advisors, research institutes etc. Indeed, if a model such as equation (3) represents a good description of an economy's motion, then the divergence of the time series in the face of very slight differences in the initial values (and no economist is able to provide current values of relevant economic values with an absolute precision) makes mid- and long-term predictions impossible. However, there does not exist any real reason why a simple model such as equation (3) 

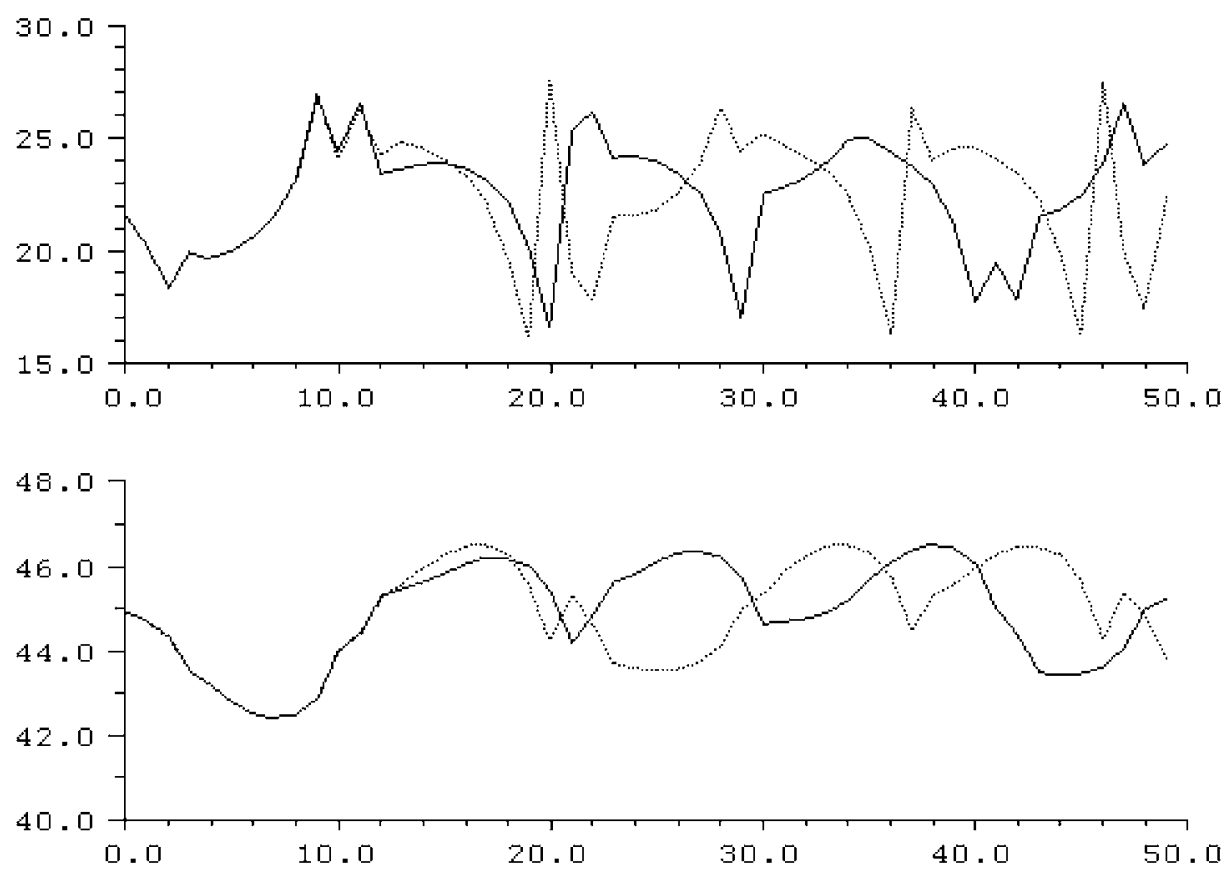

Figure 3. Two time series with slightly different initial values ( $Y$ versus time (upper plot), K versus time (lower plot))

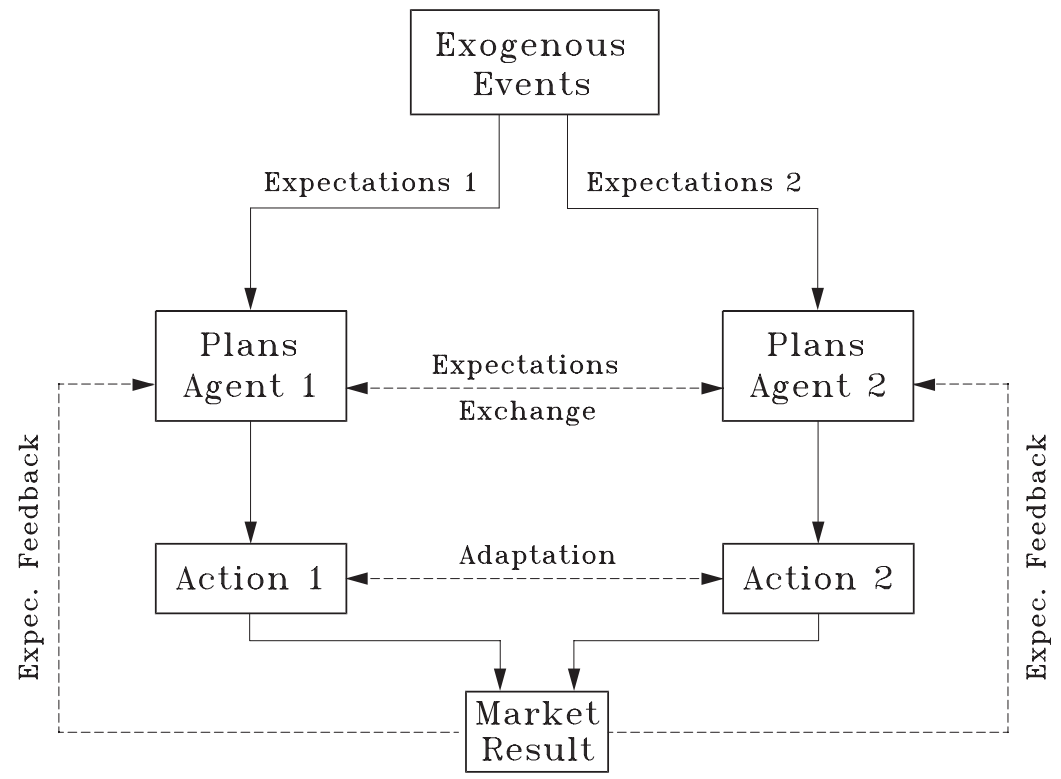

Figure 4. An example of an economy as a feedback system 
should constitute a proper description of the motion of an economy. The rest of this paper is devoted to the question of whether a model such as equation (3), which was constructed in the light of the classical/neoclassical tradition, is indeed suited to characterize actual economic motion.

Economic theory suffers from a specific modelling problem, which is typical for many social sciences: formal mathematical dynamical models may represent very simple theoretical scenarios or they might reflect complicated formal relations. On the other hand, a formal model can result either in very simple or in complex dynamics. Thus, there exist - in principle - four different modelling strategies.

(i) Simple formal models with simple dynamical results. The classical and neoclassical models mentioned above belong to this class. Since the idea of an equilibrium both in the economic sense of identical demand and supply in specific markets and in the sense of a fixed point of a dynamical system was at the centre of most theoretical reflections, simple models of market dynamics with a stable fixed point as well as models of growth dynamics represent examples of these scenarios. The class can be extended if one is willing to call business cycle models consisting of, say, two behavioural functions and appropriate definitions, a simple model, and the resulting dynamics with a closed regular orbit a simple result. The Kaldor model (equations (1) or (2)) mentioned above actually belonged to this class because the goal was to construct a simple (nonlinear) business-cycle model with regular results.

(ii) Simple formal models with complicated dynamical results. In a practical manner, the term 'complicated result' should be reserved at this place for chaotic dynamics (according to one of its various definitions) and for catastrophe theory. The title 'Simple Mathematical Models with Very Complicated Dynamics' of R. May's renowned 1976 paper $^{15}$ describes this scenario at best. Unfortunately, most economic models describe the behaviour of economic agents. There seem to exist only two possibilities: either the agents are dull, do not care about the dynamic results of their behaviour and do not change this behaviour, or the model represents an unsatisfying specification of actual economic life. The above-mentioned Kaldor model with the calculated chaotic dynamics belongs to this class because investors and consumers do not change their behaviour when they encounter chaotic motion.

(iii) Complicated formal models with simple dynamical results. The history of economic theory is full of examples with - mostly highdimensional - economic models, which turn out to generate simple 
dynamics, indeed. However, when a theorist observes simple dynamics in empirical data then the question remains why the result is not generated with simple models according to (i). One justification for this approach consists of the exciting question of how complicated a model might maximally be in order to possess still simple dynamics.

(iv) Complicated formal models with complicated dynamical results. This case appears to be the really interesting case because agents can be assumed to change their behaviour when they observe complicated dynamics. A few examples for this case will be described in the rest of the paper, although no further theoretic models will be discussed in detail.

\section{Complexity in actual economic time series?}

The complexity phenomenon in cases (ii) and (iv) mentioned above can actually be of interest for an economic theorist only when studies uncover that actual empirical time series are indeed characterized by chaotic or other complicated processes. (The case that a model with the chaos property should be dispensed or that the allowed parameter space in these models should be limited should not be followed here.)

Indeed, empirical econometric tests for the existence of chaos (mainly with the help of non-parametric statistics) were performed in the 1980s. One reason for this interest in chaotic actual time series was the hope that with the knowledge of the existence of chaos in, say, financial markets' data, higher profits could be achieved when someone knew about the existence of a deterministic nonlinear system and others still believed that stochastic influences dominated.

The results were ambiguous. Macroeconomic data (such as GDP, unemployment rates, price indices etc) showed only small evidence for the presence of chaos. Other series, such as stock market prices provided some hints for the presence of the phenomena. These relatively poor results led to a giving-up of most studies in the late 1990s.

It is possible to mention at least two explanations for this failure in attempts to detect chaos in actual time series.

(i) The requirement of large data sets: most non-parametric tests, like the determination of correlation dimensions, entropies etc, actually require the availability of infinitely large data sets or at least very large sets. Macroeconomic data are usually available in the form of annual or maximally quarterly data. Many financial markets change much quicker, and stock market prices nowadays are published every few seconds in most important financial markets. However, financial data are highly structured with Monday effects, ultimo effects, 
the publication date of balances and so on. Since most non-parametric instruments test for the presence of structure, these mentioned structures must be eliminated from the data before the instruments are applied. This filtering process does not seem to work properly and is - to some degree- arbitrary. Chaos tests are therefore highly unreliable.

(ii) The above-mentioned empirical tests declare a time series as chaotic when, for example, the correlation dimension is fractal and changes in a specific way for a low number of dimensions. A positive chaos test then implies that the generating dynamical system is lowdimensional as well. In modern economies with a huge number of variables and very many stochastic influences, this implication does not seem to be appropriate, although the correlation dimension can provide hints on whether an actual series is dominated by deterministic or stochastic influences.

Other researchers - mainly from other disciplines - still insist on the fractal nature of empirical economic data, e.g. in financial markets (cf. Mandelbrot ${ }^{9}$ ).

\section{Complexity in real economic life}

The following statement might sound strange or at least unexpected. Physics and other so-called 'hard' sciences live in a rather simple theoretical academic environment: most (or almost a majority hereof) 'laws' relevant for everyday experience seem to be known to all experts in their fields. Probably nobody will deny the importance of the Navier-Stokes equation for weather forecasts or the relevance of the classical astronomical results for the motion of earth-near celestial bodies and attempts to perform missions to other planets. While the properties of the underlying, assumed dynamical systems might occasionally be unclear (at least since the appearance of chaos phenomena), the governing dynamical systems themselves usually keep being undisputed.

Economics is different. Economic environments are permanently changing due to innovations, changing tastes etc. Political ideologies may find their routes to academic convictions (and vice versa). The important message stemming from this insight reads: there never will exist something like 'eternal' laws in economics comparable to the laws known from, say, classical mechanics for everyday experience. The following will be devoted to a few prominent examples representing this particular property of economic theorizing.

\section{Real economic life as a permanent nonlinear feedback system}

Many examples of mechanical physical systems, such as a ball wandering in a bowl, the motion of a suspended and finally released technical spring, the 
reaction of a particular material under the influence of a global heating of its environment, etc, usually represent simple installations without any feedback processes, i.e. the environment does not react to the influence this environment possesses on the installation. Other examples encompass feedback processes in the form of weakening the original external force during its impact on the installation. The case of an amplification of the original impetus after its meeting with the installation can often be found in other fields of the natural sciences.

Since economics aims at describing the behaviour of actual, living human beings, feedback processes have to be seen in a different light. Although it is actually trivial to stress that individuals react to changes in their (here: economic) environment in their own particular manner, it is a useful exercise to emphasize a few essential differences between simple physical processes and human agents living in an interdependent economic world.

In a stationary economic scenario with inherited processes that are repeated at every relevant interval (say, in harvesting, producing house wares, etc) and without the presence of major innovations asking for adaptation, individuals probably behave according to common, well-established rules. Usually, it will not be considered mandatory to reflect permanently about the behavioural processes that might have proved being optimal in the light of the historical experience. Although superficial, such a picture might describe ancient societies more or less sufficiently well.

As soon as the environment changes, this conception can change as well (changes such as environmental catastrophes will be ignored in the following). Innovations in the form of new products, new production processes or changes in the institutional framework in which activities take place might require that economic actors adapt their individual behaviour to these changes in order to perform 'optimally' in this new environment. When such innovation eras are restricted to visible time spans during which daily experiences converged to a new stationary scenario, the same qualitative description of the particular economy may be appropriate as before. When innovations represent a permanent disturbance in a succession of stationary environments the overall picture changes (see Figure 4 for a schematic representation of the following scenario).

In order to stress the role of innovations once again, assume that a more or less permanent flow of innovations occurs in an exemplary artificial economy consisting only of two economic agents (actors). Assume further that these permanent innovations in the forms mentioned above occur as exogenous events, i.e. the innovations do not result as the efforts of the two considered agents. Agents who are familiar with their established environment have to form expectations about the relevance of the innovation for their own future behaviour. These expectations might differ, either due to mental differences or different economic conditions under which the agents perform. Together with their inherited actions the expectations about the influence of innovations will influence the planned 
behaviour of the agents during the next occasion, say the next market day. Before an action really takes place, the agents might consider it useful to form expectations about other agents' expectations and appropriate plans. An 'expectations exchange process' might take place at this moment (with the possibility of an infinite regress in this adaptation process).

Provided that this adaptation process indeed ends in eventually performed actions (say the announcement of demand and supply quantities in a certain market), the agents might realize that their planned actions are incompatible, e.g. that supply is larger than demand. In that case, the agents might change their actions (not necessarily their plans) in order to achieve a market result. This market result, in the form of, for example, new prices and new information about the market size, can initiate another feedback process that can influence the plans of the agents for the next market round. These plans for the near future might then additionally be influenced by another set of exogenously determined innovations.

This seemingly trivial example of an economic feedback process represents a drastic difference between processes in the inanimate natural world and the behaviour of living beings because the latter can learn in order to react to changes in their environments in particular ways. When economic agents are - in principle able to react to changes in their environment by changing their individual (or, occasionally, collective) behaviour, the motion of an economy obviously cannot be described anymore by processes formally known from classical physics. As was mentioned above, the scientific aim for many - if not most - classical and neoclassical authors consisted of describing the motion of an economy in the same way as physicists modelled the motion of inanimate entities. Learning processes therefore represent a particular challenge for economic dynamics.

This simple insight was not shared by many economists even until recently. In the major contributions of John Maynard Keynes ${ }^{10}$ dealing with the possibilities for influencing an economy suffering from unemployment and low national product, it was assumed that private economic actors do not anticipate the activities of governments, but react to effective changes in their financial conditions when, say, taxes are increased. Changes in goods prices as the result of governmental activities did not initiate feedback processes. Even in particular theories of the dynamic behaviour of financial markets it was assumed that all individuals actually are informed about everything ${ }^{11}$ and that anticipations of the behaviour of others do not play a crucial role.

Another extremely simplistic way to circumvent modelling difficulties of these feedback processes consists of assuming that all individuals actually are informed about everything, including the expectations of other actors. In such an assumed framework, economic actors can - in principle - understand the actions of all agents in every step and can therefore 'solve' the scenario in order to determine 
a 'dynamical system', which provides full information (abstracting from pure stochastic and unforeseeable influences) about the motion of the economy. This is the approach followed by the so-called 'Rational Expectations' school, still popular in many macroeconomic studies today. A variant of this Rational Expectations approach will briefly be discussed in the next section.

\section{Goodhart's law as an example of a macroeconomic feedback process}

While the Rational Expectations approach was originally developed for a certain view on particular single markets, its popularity during the last 30 years is due to macroeconomic applications. If, in an extremely simple set-up each agent is informed about the economic environment and the expectations and planned actions of all other agents and if the behaviour of a government is known up to a random term (which cannot be foreseen) then every attempt of a government to control an economy is doomed to failure, because the intended result was already foreseen by the private public (up to the stochastic random term) by solving a certain 'true' model of the economy shared by everybody (including academics). According to this logic, a government can control an economy only in the bizarre scenario in which its planned actions are absolutely secret so that they cannot be foreseen by the public.

A particular example of such a scenario is occasionally connected with the name of a former chief executive of the Bank of England, C.A. Goodhart. Goodhart ${ }^{12}$ - as do many central bankers around the world - believed in the idea described above that private agents were able to anticipate the results of the activities of institutions such as central banks or governments because - typically - government activities are known to the public in advance in democratic societies (or can at least be expected as far as central bank activities are concerned). When private agents are well-aware of the functioning of an economy and when they are indeed wellinformed about the planned actions of these central institutions, the planned activities might result in higher goods prices, higher wages etc, in the case of expansive policies, but might not influence real entities such as gross domestic product, employment figures, and so on. In very simple theoretical frames, these perfect anticipations of political actions can result in so-called 'policy ineffectiveness' situations. Only if the public is uninformed about planned political activities and is actually completely surprised by these measures, can the economic activities of the central institutions be effective.

Goodhart's publications are written in within this debate on policy ineffectiveness. However, his name is connected with a popular interpretation of the ineffectiveness debate.

Imagine an economy in which agents are not well-informed about each and every thing and that they do not precisely know how an economy functions. Imagine further that a serious institution is using a questionnaire about the 
economic behaviour of private agents. At the very moment of the publication of these empirical investigations, the agents who took part in the questionnaire might change their attitudes, beliefs, behaviour, etc, after having learned about the behaviour of others. That is, the publication of empirical findings about the economic behaviour of others might change one's own economic behaviour. Goodhart's law - in this interpretation - states that an empirical finding might turn out to be wrong in the near future because private agents might have changed their behaviour after the first publication of an empirical investigation. Of course, these effects do not represent a universal scenario. Not all empirical findings influence the personal behaviour of private agents. As soon as the benefits, profits, etc, of private agents are directly influenced by the behaviour of others, new data might, however, very well affect the behaviour of individuals. Another feedback effect occurs in such a scenario because the published data depend - to a certainly minor degree - on the actions performed by the considered private agent him or herself.

\section{Complete versus bounded rationality}

Starting with the above-mentioned contributions by the neoclassical pioneers such as Menger or Marshall, every attempt was made to model economic actions as the consequences of so-called 'rational' agents. Assume that the following prerequisites are fulfilled: (i) full information about each and every thing that is relevant for the agents' actions; (ii) the ability of the agents to process this information and to perform adequate actions; and (iii) the presence of a harmonic coordination procedure allowing for the fulfilment of the planned actions of agents who anticipated an 'equilibrium scenario'. This neoclassical world-view depicts a stage view that indeed reminds us of the renowned classical statements in classical physics.

Items (i) and (ii) appear to be the most important candidates for a critique (a few remarks on item (iii) can be found in the following). Neoclassical authors living in the 19th century encountered a certainly simpler and slower changing economic environment than an agent living in recent times. However, the assumption of full information (not only about an objective environment but also about the beliefs and planned actions of other agents) is - to say the least heroic. In 'neoclassical' as well as in recent times, agents simply cannot possess all the information necessary for their (maybe everyday) decisions about appropriate actions. Even if they basically had access to all relevant information, the human inability to process large amounts of information would deprive agents of applying appropriate maximization procedures (note that the set of parameters and variables have to be determined in advance, even in the days of supercomputers). The problem of what a target function should look like is another important topic in criticizing neoclassical economics. 
Simon ${ }^{13}$ and others have criticized the neoclassical paradigm rather intensively and emphasized the impossibility of living humans of being able to collect and process full information about each and every thing. Instead of the postulate of 'rational' agents, the importance of a so-called 'bounded rationality' was emphasized in cases when the mentioned prerequisites of fully rational agents cannot be met. The relevant question remains: what are private agents actually doing when they have to decide on consumer demand, goods production etc, but do not possess all information necessary for reaching optimal decisions (i.e. results that usually represent maxima of particular target functions)? The following items appear as the relevant ones.

(1) Agents (including governments and central banks) often use 'rules of thumb'. When, say, a producer does not fully know his production possibilities or is not completely informed about the demand for his goods, he can then decide on applying vague decision criteria. When he just has entered a market, it might be difficult to find such a rule of thumb. Repeating a decision against the background of a rather unchanged environment might have demonstrated that the rule is possibly not too bad. The rule can then imply a 'satisficing' behaviour in the sense that the agents know that they do not know about each and every thing but that the rule-of-thumb based decision leads to economic results that can be improved only with significant effort, because the information necessary for a revision might not be available. As mentioned above, even if full information is basically available with high costs, mental restrictions might forbid processing this information. It can, however, not be excluded that applying rules of thumb may result in unsuccessful decisions. An agent might then switch to other rules or might leave the market.

(2) Agents who enter new markets or who have been unsuccessful when they applied their former rules might attempt to imitate other agents in similar environments. If, for example, a producer of a particular good had chosen a wrong price policy in the past, he might chose similar prices to his competitors, although there might exist factual room for lower or higher prices with the basic possibility of higher profits. Imitation is probably also a relevant phenomenon in the competition process between private banks and/or between central banks in an international context.

In any case, the application of rules, rather than performing attempts to solve full optimization problems, speeds up the decision processes and is cheaper, because the collection of information is almost always costly. Unfortunately, while these basic insights are appreciated by many recent economists and authors of older 
contributions, such as Veblen, ${ }^{14}$ mainstream economic theory never really adopted the concept of bounded rationality and therefore ignored one of the most important appearances of CERL scenarios.

One of the reasons why the concept of bounded rationality is still so uncommon in standard economic theory might be because it is rather difficult to model a vague, uncertain world that is not always characterized by simple, eternally valid functional relations. Usually, real economic life is open for new production processes, new products, changing tastes - implying a permanently present need for agents to gather new information and orient their actions at this changing environment (or their beliefs about this environment). The so-called 'Experimental Economics' where living persons perform tests in computer laboratories represent a fruitful field for the study of basically open sets of choices among possible alternatives in production and consumption decisions. It might also be possible to develop something like a 'dynamic' decision and action scenario in the light of the concept of bounded-rationality.

It might seem easy to claim the absence of full rationality or the presence of bounded rationality in academic reflections about real economic life, but then it might appear difficult to find relevant economic examples in the recent past and present. However, it is actually relatively easy to encounter such examples.

Traditionally, financial markets are considered as examples of markets in which agents - mostly professional brokers - are more or less fully informed and are able to delve into the material and are able to process the information in an optimal way. As such, financial markets are often considered as a prototype example of markets with fully rational agents. Financial markets are therefore often called 'efficient' markets. What has often been called a 'financial crisis' starting sometime around the end of 2007/beginning of 2008 - can be called a counterexample to this view. While many experts see the origins of the crisis more than 30-40 years ago when relevant institutional (also international) arrangements were made, the dramatic effects started after the collapse of a few US banks, probably due to mismanagement, with the result that - mainly due to so-called 'sub-prime loans', i.e. loan contracts that were sold by original creditors to other companies in the market - the crisis of a few banks created a collapse of the US housing market and further liquidity problems of other banks. The interesting feature of the spread of the crisis is that seemingly even professional financial agents were rather unconscious about the intertwinement of diverse businesses in the markets. A few observers even claim that many agents were uninformed, at least about the details of many new financial constructs, such as variants of hedge funds, options, derivatives etc. In addition, the extremely high volatility of share prices during the crisis might indicate that financial agents are characterized by a sort of herd behaviour, i.e. they adopt the behaviour of others. Gathering new information and processing it has appeared as being much too 
time consuming in the light of rapid changes - within hours - in financial markets. In this sense, the crisis seems to be a dramatic example of bounded rationality in an entire market.

The (possible) dimension problem in dynamic economic scenarios

Most examples of dynamical systems (here considered for a continuous time concept) in the natural sciences represent functional relations between the current values of the variables relevant for a particular problem at a particular point (or different points) in time and the change of these variables in the form of welldefined systems of differential equations, such as

$$
\frac{\mathrm{d} \mathbf{x}}{\mathrm{d} t}=\mathbf{f}(\mathbf{x}), \quad \mathbf{x} \in \mathbf{R}^{n}
$$

with $\mathbf{f}(\cdot)$ as an unchanging vector function for the considered time horizon. The dimension $n$ of the vector $\mathbf{x}=\left(x_{1}, x_{2}, \ldots, x_{n}\right)$ of state variables is usually assumed as being constant.

Dynamic economic theory might encounter a particular problem. Assume that the motion of the variables in a set of markets or the entire economy can be described by a dynamical system like equation (4) with $\mathbf{x}$ denoting, for example, the vector of products produced in various sectors. The amount of goods produced in each sector is therefore assumed to depend on the production of other goods in other sectors.

However, economic dynamical systems can hardly be claimed to persist forever. Innovations in the form of the introduction of new products, new production processes, the opening of new markets, institutional changes which, for example, result in free or closed market access etc, delimit the validity of a system (4) which might then be defined only for a certain time window, say, January 2008 to March 2008. Such a time window can then be called a stationary valid glimpse at the economic world in which no innovations (or, better, noticeable innovations) take place.

Innovations introduced during the next time period (which is defined by the occurrence of these innovations) imply different functional forms and might change the dimension of the dynamical system. Assume that the new system reads

$$
\frac{\mathrm{d} \mathbf{y}}{\mathrm{d} t}=\mathbf{g}(\mathbf{y}), \quad \mathbf{y} \in \mathbf{R}^{n+m}
$$

with $\mathbf{g}$ as the new vector function and $\mathbf{y}=\left(y_{1}, y_{2}, \ldots, y_{n+m}\right)$ as the new vector of state variables. According to the specific innovation history, the number $m$ can be smaller, equal to, or larger than zero, indicating that new products emerge, old products disappear in the production process, or that incidentally the dimension stays constant (even in a scenario with innovative activities). 
The new dynamical system (5) can have two consequences.

(i) The considered innovations do not change the basic properties of the economy's dynamic behaviour (e.g. fixed points remain stable or unstable, oscillations persist with no drastic changes in amplitudes, or no other additional phenomena emerge). In such a case, innovations might be called 'dynamically irrelevant phenomena'.

(ii) The change in the dynamical system and its dimension might imply drastic changes in the dynamic behaviour of the time path. According to an often expressed - but actually unproved - conjecture, an increase in $n$ increases the probability of encountering complex dynamic behaviour of the system as compared with an original low-dimensional system.

If the first property holds for several time windows, the qualitative nature of an economy's dynamic behaviour can - in principle - be described by the original system (equation (4)). However, a possible change in the dynamic consequences can be detected only by analysing the modified system (equation (5)) itself. Thus, in any case, innovations and similar events imply the necessity of studying a succession of dynamical systems, each of which is defined only for a probably limited time horizon.

\section{Conclusions}

Large parts of modern mainstream economics find their roots in the particular scientific attitude towards reality, popularized in the 19th century, which can be called the 'mechanistic world view'. While other disciplines, such as biology, chemistry or the newly emerging sociology, developed their own scientific methods with varying degrees of determinateness and distinctiveness, the economics of the day can be characterized as an attempt to establish well-defined mathematical forms between independent and dependent variables. Economic variables were seen as entities reacting in the same way as pressure, tension, heat, etc, to other physical variables in an environment with a given and constant parameter set. Either by pure assumption or as attempts to derive individual behaviour from optimization procedures - provided that the relevant information is available and individuals are mentally and technically able to optimize at all - the behaviour of most economic subsystems, such as households, firms, governments or entire markets and national and global economies, is typically modelled as a deterministic set of well-defined mathematical functions.

Much in the same way as classical mechanics, economics concentrated on the possibility of equilibria in the sense of resting points in time. Motion was conceived as the transition from one resting point to another in response to changes in the economic environment. The transient phase itself was only seldom 
investigated. Only in particular economic sub-disciplines, such as business-cycle theory, in which oscillatory behaviour was intentionally modelled, or in growth theory, where trend increases of, say, the national product were emphasized, was the stationary equilibrium concept abandoned.

Ironically, the popularization of the chaos phenomenon in many nonlinear deterministic dynamical systems (in many disciplines) also uncovered that chaotic motion represents a least a mathematical possibility in traditional dynamic economic models. Many nonlinearities responsible for the presence of unexpected phenomena such as the SDIC or completely aperiodic motion either already existed in a variety of traditional economic models or can easily be assumed as a certain extent in a broader class of well-accepted economic approaches. It therefore seems as if the chaos phenomenon at least represents a common property of many economic models. Unfortunately, this chaos phenomenon contradicts a fundamental intent of economic theory and a consecutive economic policy, namely the attempt to forecast future events with a possibly high precision. For decades, economists were as optimistic as meteorologists that, with the help of additional measuring devices, bigger computers and theoretical refinements, the quality of forecasting, even for longer time spans, could be improved. However, even if actual economies indeed behave according to the functionally simple, low-dimensional models for which the existence of chaos has been established, not very much forecasting success can be expected.

Early non-parametric statistical work on the presence of chaos in actual time series in macroeconomic and financial markets data provided some academic hope that more insights into the dynamics of extremely important everyday phenomena could be yielded. It was indeed possible to detect measures such as positive Lyapunov exponents, fractal correlation dimensions, etc, but it became obvious relatively early that these measures are more or less meaningless in the light of actually extremely high-dimensional systems. The fact that the standard chaos indicators provide hints for the presence of relevant nonlinearities somewhere in the system is of no help as long as the starting points for controlling chaos are unknown.

Actual economies cannot be described by fixed and deterministic laws of motion. While the presence of longer-lasting functional forms and institutions often cannot be denied, most modern economies are characterized by vanishing or emerging variables, changing dynamical systems with possibly changing dimensions and probably non-neglectable external stochastic influences. Altogether, since nobody can claim to oversee all singular elements with their particular properties and their interactions with other entities, this gives a reason to presume that actual economies represent some of the most complex dynamical systems of all. The depiction of economies (or sub-systems) in the form of low-dimensional deterministic systems (as in classical economics) therefore does not appear as a completely appropriate task. The real challenge for economic theory consists of attempts to find reliable 
descriptions (in formal or informal terms) of particular economic phenomena with the consciousness that a full understanding of economies as complex systems will probably never be possible.

\section{References}

1. J. H. Holland (2006) Studying complex adaptive systems. Journal of System Sciences and Complexity, 19, 1-8.

2. C. Menger (1871) Grundsätze der Volkswirtschaftslehre. Reprinted in: F. A. Hayek (ed.) (1969) Carl Menger. Gesammelte Werke, Band I (Tübingen: J.C.B. Mohr).

3. L. Walras (1874) Élements d'Économie Politique Pure (Lausanne: L. Corbaz).

4. F. Y. Edgeworth (1881) Mathematical Psychics (London: C. Kegan Paul).

5. W. S. Jevons (1871) The Theory of Political Economy (London: Macmillan).

6. A. Marshall (1890) Principles of Economics, Volume I (London: Macmillan).

7. R. Herrmann (1985) Stability and chaos in a Kaldor-type model. Discussion Paper, Department of Economics, University of Göttingen, 22, 1-16.

8. H.-W. Lorenz (1992) Nonlinear Dynamical Economics and Chaotic Motion, 2nd edn (Berlin, Heidelberg, New York: Springer-Verlag).

9. B. Mandelbrot (2004) The (mis)Behavior of Markets - A Fractal View of Risk, Ruin and Reward (New York: Basic Books).

10. J. M. Keynes (1936) The General Theory of Employment, Interest, and Money (London: Macmillan).

11. W. B. Arthur (1995) Complexity in economics and financial markets. Complexity, 1, 20-25.

12. C. A. E. Goodhart (1975) Monetary relationships: a view from Threadneedle Street. In: Monetary Economics, Vol. I (Reserve Bank of Australia).

13. H. A. Simon (1955) A behavioral model of rational choice. Quarterly Journal of Economics, 69, 99-118.

14. T. Veblen (1899) The Theory of the Leisure Class (New York: Macmillan).

15. R. M. May (1976) Simple Mathematical models with very complicated dynamics. Nature, 261, 459-467.

\section{About the Author}

H.-W. Lorenz started his studies in economics at the Georg-August-University in Göttingen in 1971 where he graduated in 1977. His $\mathrm{PhD}$ thesis was concerned with a problem in non-Walrasian macroeconomic dynamics, namely the problem of simultaneous price and quantity adjustments in disequilibrium scenarios with rationing. After longer sabbatical stays as a visiting professor at the University of California at Berkeley and the University of Southern California in Los Angeles as a visiting professor he became familiar with the emerging field of chaotic motion in theoretical models and actual empirical time series in economic dynamics. His efforts were summarized in his (German) habilitation thesis in 1991 in a broader philosophical context. He is currently working as a full professor of economics at the Friedrich-Schiller-University in Jena, Germany. 